\title{
One-stage intracardiac repair in combination with external stenting of the trachea and right bronchus for tetralogy of Fallot with an absent pulmonary valve and tracheobronchomalacia
}

\author{
Takahiko Sakamoto, MD, ${ }^{a}$ Yuzo Nagase, MD, ${ }^{c}$ Hisaya Hasegawa, MD, ${ }^{d}$ \\ Toshiharu Shin'oka, MD, ${ }^{a}$ Hirofumi Tomimatsu, MD, ${ }^{b}$ and \\ Hiromi Kurosawa, MD, ${ }^{a}$ Tokyo and Chiba, Japan
}

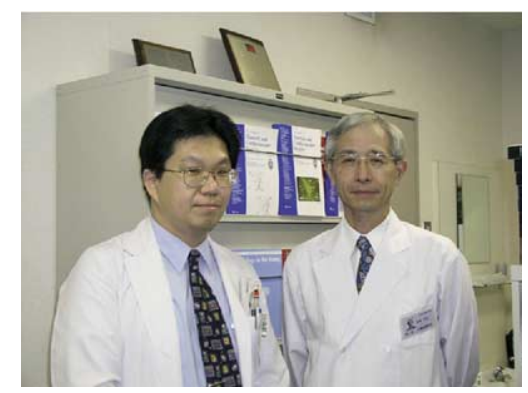

Drs Sakamoto and Kurosawa

\section{Clinical Summary}

The patient was noted to be cyanotic immediately after birth. Echocardiography revealed tetralogy of Fallot with an absent pulmonary valve. He showed severe respiratory dysfunction 1 month later and was intubated because of the appearance of apnea. Cardiac catheterization showed left ventricular end-diastolic volume of $88 \%$ of normal, left ventricular ejection fraction of $58 \%$, right ventricular end-diastolic volume of $188 \%$ of normal, right ventricular ejection fraction of $53 \%$, pulmonary/systemic flow ratio of 2.7 , and pulmonary artery (PA) index of $1290 \mathrm{~mm} / \mathrm{m}^{2}$. Repeated inadequate ventilation required a careful examination, and bronchoscopy elucidated a narrowing of the trachea and a flat shape of the right bronchus with granulations. High positive endexpiratory pressure ventilation therapy for 2 weeks under deep sedation was scheduled to cure the granulations, and definitive surgical intervention was performed at the age of 3 months.

Through a median sternotomy, the trachea and right bronchus were dissected carefully. Ringed expanded polytetrafluoroethylene (ePTFE) grafts $(\varphi 14 \mathrm{~mm})$ were trimmed to a circumference of 12 $\mathrm{mm}$. They were passed around the trachea and the right main bronchus and sutured with no. 6-0 polypropylene sutures. The membranous portions were also covered with a piece of ringed ePTFE patch. Bronchoscopy revealed that the inner lumina of the trachea and right bronchus became round after the procedure (Figure 1).

Cardiopulmonary bypass was then started, with direct cannulations of the ascending aorta and the superior vena cava and inferior vena cava. After cardiac arrest, the abnormal musculature of the right ventricular outflow tract was resected through a right

\footnotetext{
From the Departments of Cardiovascular Surgery ${ }^{\mathrm{a}}$ and Pediatric Cardiology, The Heart Institute of Japan, Tokyo Women's Medical University, Tokyo, Japan, and the Divisions of Cardiovascular Surgery ${ }^{\mathrm{c}}$ and Neonatology, ${ }^{\mathrm{d}}$ Matsudo Municipal Hospital, Chiba, Japan.

Received for publication July 2, 2005; accepted for publication Aug 3, 2005.

Address for reprints: Takahiko Sakamoto, MD, Division of Cardiovascular Surgery, Kanagawa Children's Medical Center, 2-138-4, Mutsukawa, Minami-ku, Yokohama-city, Kanagawa 232-8555, Japan (E-mail: takasakamoto@yahoo.co.jp).

J Thorac Cardiovasc Surg 2005;130:1717-8

$0022-5223 / \$ 30.00$

Copyright (C) 2005 by The American Association for Thoracic Surgery doi:10.1016/j.jtcvs.2005.08.006
}

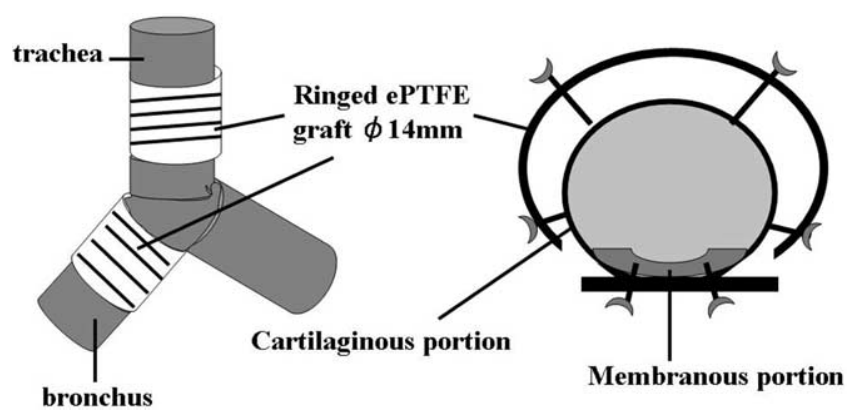

Figure 1. Diagram of operative technique. Ringed ePTFE grafts $(\varphi 14 \mathrm{~mm})$ were trimmed to a circumference of $12 \mathrm{~mm}$. They were passed around the trachea and the right main bronchus and sutured with no. 6-0 polypropylene sutures.

ventricular incision. The ventricular septal defect was a perimembranous outlet type $(10 \times 10 \mathrm{~mm})$, and it was closed with an ePTFE patch through a right atriotomy. Pulmonary arteriorrhaphy was performed by excising a generous portion of the anterior walls of the main, right, and left PAs. The right ventricular outflow tract reconstruction was done with a handmade monocuspid pericardial patch. Weaning from cardiopulmonary bypass was uneventful, and the tracheal tube was removed on postoperative day 8 . Postoperative cardiac catheterization data at 1 year showed a systemic flow of $4.1 \mathrm{~L} \cdot \mathrm{min}^{-1} \cdot \mathrm{m}^{-2}$ and a central venous pressure of $5 \mathrm{~mm} \mathrm{Hg}$. Three-dimensional computed tomographic scans showed an excellent status of external stenting for the trachea and right bronchus (Figure 2).

\section{Discussion}

Tetralogy of Fallot with an absent pulmonary valve is a critical congenital heart defect. ${ }^{1}$ We have performed 19 surgical operations for this defect over the last 20 years. Eight (42\%) patients were younger than 1 year, and the appearance of respiratory disturbances in the neonatal or infantile period was associated with a poor prognosis. On the other hand, the long-term outcome remains unknown, even if the definitive operation was successful. Progressive enlargement of the PA and the resultant compression of the trachea or bronchus are serious problems in the long-term period. ${ }^{2,3}$ In our experience one patient required surgical intervention, which included an external stent against the secondary bronchomalacia caused by pro- 


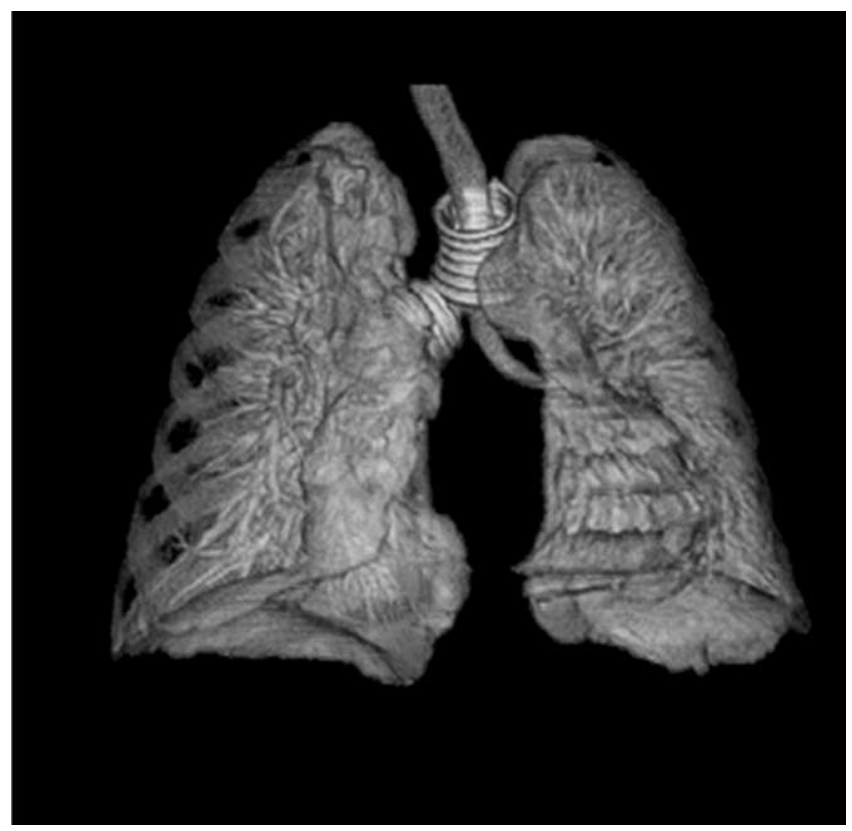

Figure 2. Postoperative 3-dimensional computed tomographic scan.

gressive enlargement of the right PA after definitive surgical intervention.

Recent developments in bronchoscopy have contributed to an early diagnosis of tracheobronchomalacia. However, this condition is classified as primary or secondary malacia, and optimal surgical treatment is controversial. Under these circumstances, treatments such as aortopexy, internal stenting, or prolonged high positive end-expiratory pressure ventilation, have been attempted, although satisfactory results have not been obtained. We started the external stenting technique using a ringed ePTFE graft in 1997, when we unexpectedly noticed that $\mathrm{Hagl}$ and associates ${ }^{4}$ reported their first experience of a very similar technique between 1992 and 1995 . We placed a separate graft and patch around the respiratory tract (it is important to suture the first graft on the border region between cartilaginous portion and membranous portion), whereas Hagl and associates sutured the ePTFE graft completely closed. We believe that our technique has the potential of growth compared with that of Hagl and colleagues, although growth might not be needed if the graft is of sufficient size ( $>14 \mathrm{~mm}$ in diameter).

In the current case single-stage intracardiac repair with external stenting was performed. The advantages of this procedure are (1) to establish both normal hemodynamics and relief of the respiratory disturbance, (2) to be effective for cardiac anomalies in combination with primary tracheobronchomalacia, and (3) to prevent airway narrowing from the progressive enlargement of the PA in the long-term period. On the other hand, there are some disadvantages to this procedure. The graft might become weak because of infection. When an infection occurs, the stent graft should be removed, and the patient should be returned to conservative treatment. Also, there is a concern that the circumferential nature of the graft might lead to devascularization of the trachea, compounding the problem of cartilage loss. However, because of its great advantages, we believe that the current external stent technique is useful for cardiac patients with associated with tracheobronchomalacia, although careful observation should be performed. ${ }^{5}$

\section{References}

1. Castaneda AR, Jonas RA, Mayer JE, Hanley FL. Cardiac surgery of the neonate and infant. Philadelphia: WB Saunders; 1994. p. 232-3.

2. Rabinovitch M, Grady S, David I, Van Praagh R, Sauer U, Buhlmeyer K, Castaneda AR, Reid L. Compression of intrapulmonary bronchi by abnormally branching pulmonary arteries associated with absent pulmonary valves. Am J Cardiol. 1982;50:804-13.

3. Momma K, Ando M, Takao A. Fetal cardiac morphology of tetralogy of Fallot with absent pulmonary valve in the rat. Circulation. 1990;82:1343-51.

4. Hagl S, Jakob H, Sebening C, van Bodegom P, Schmidt K, Zilow E, et al. External stabilization of long-segment tracheobronchomalacia guided by intraoperative bronchoscopy. Ann Thorac Surg. 1997;64:1412-20.

5. Wells WJ, Hussain NS, Wood JC. Stenting of the mainstem bronchus in children: a word of caution. Ann Thorac Surg. 2004;77:1420-2. 